\title{
Genetic Causes of Generalized Epilepsies
}

\author{
Ingo Helbig, $\mathrm{MD}^{1,2}$ \\ ${ }^{1}$ Division of Neurology, The Children's Hospital of Philadelphia, \\ Philadelphia, Pennsylvania \\ 2 Department of Neuropediatrics, Christian-Albrechts-Univerisity of \\ Kiel and University Medical Center Schleswig-Holstein (UKSH), \\ Kiel, Germany
}

Semin Neurol 2015;35:288-292.

\begin{abstract}
Address for correspondence Ingo Helbig, MD, Division of Neurology, The Children's Hospital of Philadelphia, 34th St and Civic Center Blvd, Philadelphia, PA 19104 (e-mail: helbigi@email.chop.edu).
\end{abstract}

\begin{abstract}
Keywords

- genetic generalized epilepsy

- copy number variation

- exome sequencing

Generalized epilepsies, particularly the idiopathic or genetic generalized epilepsies (GGEs), represent some of the most common epilepsies. Clinical genetic data including family studies and twin studies provide compelling evidence for a prominent genetic impact. The first decade of the 21 st century was marked by progress in understanding the basic biology of generalized epilepsies including generalized/genetic epilepsies with febrile seizures plus (GEFS + ) and GGE through studies of large families, discovering causative mutations in SCN1A, SCN1B, GABRG2, and GABRA1. Subsequently, recurrent microdeletions at $15 q 13.3,16 p 13.11$, and $15 q 11.2$ were found to be relevant risk factors for nonfamilial GGE. Genes for epileptic encephalopathies such as SLC2A1 were rediscovered in GGE, highlighting the biological continuum between different epilepsies. Genome-wide studies examining common genetic risk factors identified common variants in SCN1A, indicating a convergence of shared pathophysiological pathways in various types of epilepsies. In the era of next-generation sequencing, however, the GGEs appear more complex than expected, and small or moderately sized studies give only a limited genetic perspective. Thus, there is a strong impetus for large collaborative investigations on an international level.
\end{abstract}

The common generalized epilepsies account for up to onethird of all epilepsies, presenting one of the most common epilepsy subtypes encountered by neurologists. ${ }^{1}$ Most patients with generalized epilepsies either present with one of three subtypes; childhood absence epilepsy (CAE), juvenile absence epilepsy (JAE), or juvenile myoclonic epilepsy (JME). These conditions, formerly referred as the idiopathic generalized epilepsies (IGEs), are characterized by an electroclinical phenotype with a combination of typical absence seizures, myoclonic seizures, and primary generalized tonic-clonic seizures. The electroencephalogram (EEG) is characterized by generalized discharges of 3 to $6 \mathrm{~Hz}$ or faster on an otherwise normal background. Neuroimaging, if performed, is usually normal, and patients have no or little cognitive impairment or neurodevelopmental comorbidities. For a clinician, these disorders are both straightforward and perplexing at the same time. On the one hand, a typical constellation of clinical seizure types and electrographic features allows for an accurate clinical diagnosis, and in many cases, treatment is successful with antiepileptic medication. On the other hand, the etiology of these conditions is enigmatic. The generalized discharges found in these conditions conceptually suggest that the underlying pathophysiology may be diffuse, and the frequent lack of neurodevelopmental comorbidities implies that the molecular pathways leading to seizures may be specific for paroxysmal hypersynchrony in the absence of more global clinical findings. This combination has made the primary generalized epilepsies a fascinating target for research.

\section{Categories and Diseases}

Despite their frequency and distinct clinical presentation, precise classification of the generalized epilepsies has often
Issue Theme Etiology of Epilepsy; Guest Editors: Philip Smith, MD, FRCP, FAcadMEd, and Rhys Thomas, BSc, MRCP, MSc, PhD
Copyright $\odot 2015$ by Thieme Medical Publishers, Inc., 333 Seventh Avenue, New York, NY 10001, USA. Tel: +1(212) 584-4662.
DOI http://dx.doi.org/ $10.1055 / \mathrm{s}-0035-1552922$. ISSN 0271-8235. 
presented a challenge, balancing the clarity of the electroclinical syndromes against the uncertainty of the underlying etiology. The most recent classification of the International League Against Epilepsy ${ }^{2}$ reinstituted the concept of electroclinical syndromes, reflecting the clinical patterns of most of the generalized epilepsies. In contrast to previous classifications, the most recent classification has abandoned the terminology "idiopathic generalized epilepsy" because of the vagueness and ambiguity of the underlying concepts. However, given the frequently observed overlap of clinical features, the League suggested referring to this group of epilepsies collectively as "genetic generalized epilepsies" (GGEs), although this terminology is not uniformly accepted. ${ }^{3}$ For the purpose of this review, generalized epilepsies are conceptualized as a combination of the GGE proper and the generalized/genetic epilepsies with febrile seizures plus $(\mathrm{GEFS}+)^{4}$ The genetics of the epileptic encephalopathiesan expanding and exciting area-will not be covered in this review with the exception of rare epilepsies that largely overlap with GGE, such as early-onset absence epilepsy (EOAE) and myoclonic astatic epilepsy (MAE).

\section{Genetic Epidemiology}

There are few neurodevelopmental disorders that provide such striking evidence for a genetic cause as the electroclinical syndromes of childhood absence epilepsy, juvenile absence epilepsy, and juvenile myoclonic epilepsy. Several twin studies, starting with the classical studies by William G. Lennox in the 1940s, suggest that the concordance in identical twins exceeds $90 \% .^{5,6}$ This, compared with the much lower concordance in nonidentical twins, suggests that on a population level, the vast majority of causative factors are genetic. This overwhelming role of genetic factors is reflected in genetic epidemiological studies, which suggest that the recurrence in siblings for CAE, JAE, and JME is higher than in other epilepsies. For example, in a recent detailed populationbased study, the standardized incidence ratio for CAE, JAE, and JME was $6.0,^{3}$ almost twice as high as for all epilepsy combined (standardized incidence ratio $[S I R]=3.3$ ).

\section{Familial Epilepsies}

The dawn of gene discovery in human epilepsies was made possible by deciphering the underlying genetic defect in large autosomal dominant families with GEFS+ and GGE. The concept of GEFS + depends upon a familial constellation of clinical symptoms, with febrile seizures as the main feature. However, in contrast to familial febrile seizures, affected individuals and/or family members have additional seizures types or epilepsy syndromes. This phenotypic constellation in families represents a spectrum, often referred to as the GEFS+ spectrum. ${ }^{4}$ The clinical recognition of the diverse GEFS+ phenotypes in a single family as the variable expression of a single genetic disease rather than random association of diverse phenotypes was the key step to identifying SCN1A, $S C N 1 B$, and GABRG2 as genes for monogenic epilepsies. ${ }^{7-12}$ $S C N 1 A$ and SCN1B code for subunits of voltage-gated sodium channels; GABRG2 is the gene for the gamma-2 subunit of the GABA-A receptor. All three findings were pivotal in establishing the channelopathy concept of human epilepsies, ${ }^{13}$ postulating ion channel alterations as the main pathological correlate of human seizure disorders (-Fig. 1).

The prominence of mutations in these genes in the rare syndrome of GEFS + is in contrast to the relative paucity of gene discoveries in the common epilepsies of GGE and febrile seizures alone: This is remarkable and not easily explained. However, after discovery of these three genes, discovery of other genes for GEFS + stagnated for more than a decade until the recent discovery of STX1B mutations in GEFS.$+{ }^{14}$ STX1B codes for syntaxin $1 \mathrm{~B}$, a critical component of the presynaptic fusion machinery. Genetic studies in large families with GGE identified mutations in GABRG2 in families with absence epilepsy as the predominant phenotype $\mathrm{e}^{11,12}$ and GABRA1 in families with juvenile myoclonic epilepsy. ${ }^{15}$ GABRA1 encodes the $\alpha-1$ subunit of the human GABA-A receptor, further adding to the prevailing channelopathy concept of human epilepsies. ${ }^{16}$ EFHC1 coding for the EF-hand domain-containing protein 1 was initially suggested as a gene for familial juvenile myoclonic epilepsy. However, recent studies cast doubt on whether the identified variants were in fact causative, given the enormous genetic variability of the EFHC1 gene $^{17}$ and the frequency of apparently causative mutations in control databases. ${ }^{18}$ In addition, the rare variants in CACNA1H, coding for a T-type calcium channel important in the thalamocortical circuitry, may predispose to GGE. ${ }^{19}$ However, this finding has not yet been confirmed in the era or high-throughput sequencing, and it is currently unclear if and to what extent genetic variation in $C A C N A 1 H$ contributes to GGE. More recently, mutations in SLC2A1 were identified in patients with rare generalized epilepsies including frequencies of up to $10 \%$ in early-onset absence epilepsy (EOAE) and myoclonic astatic epilepsy (MAE). ${ }^{20,21}$ SLC2A1 or GLUT1 codes for the major glucose transporter in the mammalian blood-brain barrier and had previously been described in patients with severe epileptic encephalopathies and hypoglycorrhachia ${ }^{22}$ and patients with paroxysmal exercise-induced dyskinesia. ${ }^{23}$ Many patients with SLC2A1-associated generalized epilepsies have familial forms, and some rare patients with otherwise typical GGE syndromes may have SLC2A1 mutations. ${ }^{24}$ This finding, in combination with the ketogenic diet as a possible treatment option, may suggest screening for GLUT1 deficiency through metabolic or genetic studies in patients with difficult-to-treat GGE.

\section{Copy Number Variations}

After almost a decade of stagnation in gene discovery in the generalized epilepsies, copy number variations (CNVs) emerged as significant risk factors for the GGE. Copy number variations or structural genomic variants are small deletions or duplications of genomic material larger than $1 \mathrm{kB} .{ }^{25}$ Given that alterations of chromosomal dosage were difficult to assess using sequencing technologies, the extent of structural genomic variability had been previously underestimated. Novel technologies that could assess the amount of structural 


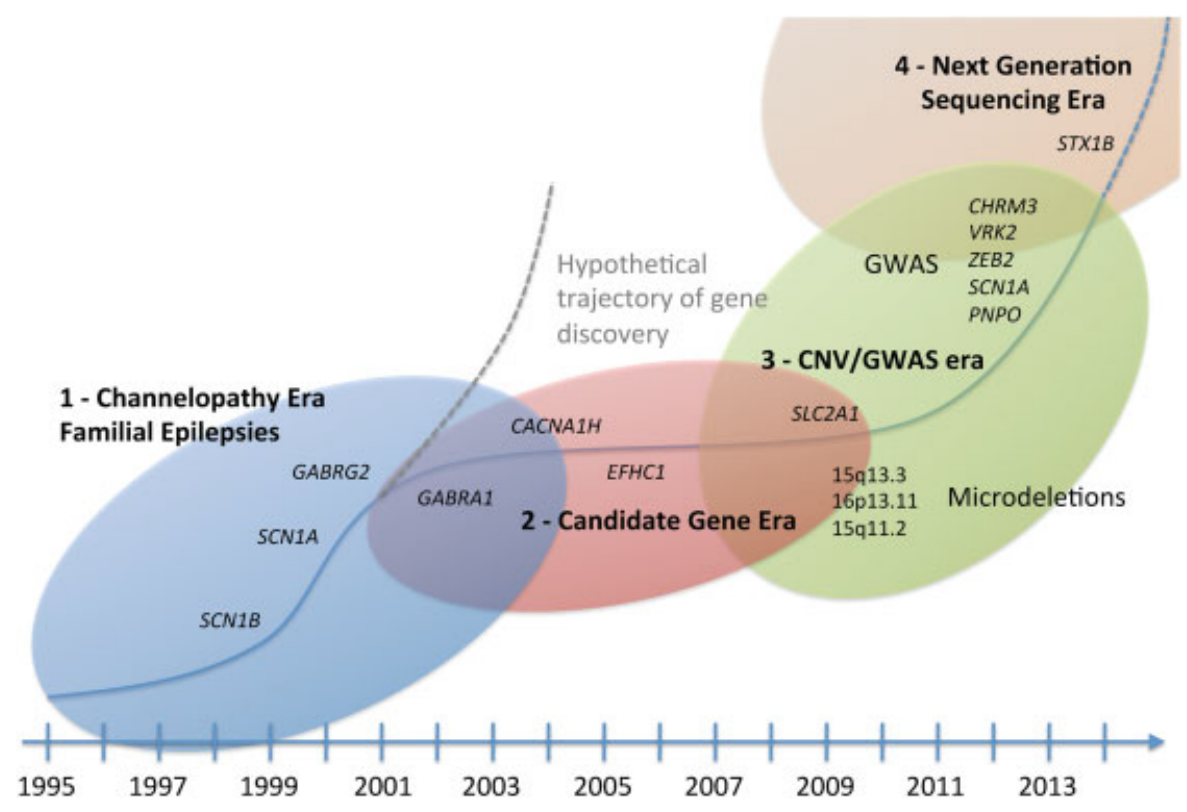

Fig. 1 Gene discovery in the generalized epilepsies. Gene discovery in the generalized epilepsies can be distinguished into four different phases: (1) The initial era of linkage studies in large families, which led to the (2) era of candidate gene sequencing when gene discovery through candidate genes did not adhere to the initially assumed trajectory of identifying explanatory ion channel genes; the (3) era of genome-wide studies including copy number variation (CNV) analysis and genome-wide association studies (GWASs); and (4) the era of massive parallel sequencing, which has only just started in the generalized epilepsies.

genomic variation on a genome-wide scale, including array comparative genomic hybridization and single nucleotide polymorphism (SNP) arrays, eventually allowed for a first insight into the unexpectedly frequent type of genetic variation from 2005 onwards. ${ }^{26}$ Analysis of large cohorts of patients with GGE showed that $1 \%$ of all patients with GGE carried a microdeletion at $15 q 13.3$, which was virtually absent in controls. ${ }^{27,28}$ Subsequently, microdeletions at 16 p13.11 and 15q11.2 were found in GGE, as well as in single individuals with deletions at $1 \mathrm{q} 21.1,16 \mathrm{p} 11.2$, and $22 \mathrm{q} 11.2 .^{29}$ All these microdeletions were previously found in other neurodevelopmental disorders including autism, intellectual disability, and schizophrenia. The common theme of these

Table 1 Genetic risk factors for the generalized epilepsies

\begin{tabular}{|l|}
\hline Autosomal dominant causes \\
\hline GEFS+ \\
\hline SCN1A, SCN1B, GABRG2, STX1B \\
\hline GGE \\
\hline GABRG2, GABRA1, EFHC1 ${ }^{\text {a }}$ \\
\hline Rare genetic risk factors \\
\hline microdeletions $15 q 13.3,16 p 13.11,15 q 11.2$ \\
\hline CACNA1H ${ }^{\text {a }}$ \\
\hline Common genetic risk factors \\
\hline CHRM3, VRK2, ZEB2, SCN1A, PNPO \\
\hline
\end{tabular}

Abbreviations: GEFS + , generalized/genetic epilepsies with febrile seizures plus; GGE, genetic generalized epilepsies.

${ }^{\mathrm{a}}$ Questionable. alterations is their genomic mechanism. These microdeletions are recurrent, arising de novo in unrelated individuals. This phenomenon is due to flanking segmental duplications serving as default breakpoints during a process referred to as nonallelic homologous recombination (NAHR) during meiosis. ${ }^{25}$ Basically, given the similarity of the flanking chromosomal regions, the replication machinery introduces deletions or duplications through unequal matching of chromosomal strands. Chromosomal regions flanked by such segmental duplications are referred to as genomic hotspots. Many known genetic syndromes including Charcot-MarieTooth syndrome, Angelman syndrome, or Di George syndrome are largely due to structural genomic variations at such loci. ${ }^{25,30,31}$ Accordingly, diseases arising from genomic hotspots are referred to as genomic disorders. Given that most genomic disorders have distinct clinical features, the discovery of genomic hotspot deletions in GGE was surprising, particularly because microdeletion carriers with GGE frequently did not have comorbid neurodevelopmental disorders. Particularly, the discovery of microdeletions at 15q13.3, $16 p 13.11$, and 15q11.2 helped to establish a novel view of the genetic architecture of GGE, emphasizing the shared genetic factors with other neurodevelopmental disorders. ${ }^{32,33} \mathrm{Col}$ lectively, it is assumed that on a population level, up to $2 \%$ to $3 \%$ of the genetic liability to GGE can be explained through these recurrent microdeletions. Although the 15q13.3 microdeletion is assumed to confirm a relatively high risk to GGE, the individual contribution of $16 \mathrm{p} 13.3$ and $15 \mathrm{q} 11.2$ microdeletions, also present in $0.5 \%$ to $1 \%$ of patients, is lower given the presence in control populations. In summary, copy number variants emerged as unexpected risk factors for generalized epilepsies, establishing the first rare genetic risk factors 
for common epilepsies. ${ }^{34}$ The analysis of copy number variants presents a first foray into the detection of genetic risk factors through genome-wide studies and already foreshadowed some of the complexities with rare genetic risk factors compared with monogenic variants. For example, despite the high odds ratio (OR) of the 15q13.3 microdeletion (OR $=68$ ), family studies frequently revealed a counterintuitive segregation pattern, characterized by an apparent lack of segregation of the variant with the familial disease phenotype. ${ }^{35}$

\section{Genome-Wide Association Studies}

Genome-wide association studies (GWASs) query the association of common genetic variants with the disease phenotype. In many disorders with a previously unknown genetic architecture, GWASs proved crucial to gain first insights into the underlying biological pathways, given that it allowed for an unbiased, hypothesis-free screen for risk factors on a genome-wide basis. ${ }^{36}$ Common variants are investigated in GWASs and are frequent in both cases and controls, usually conferring a relatively small risk, which is often insufficient to explain much of the overall disease risk and hence inadequate for genetic counseling purposes. For many neurodevelopmental disorders, however, the success of GWAS came relatively late, as unexpectedly large sample sizes were needed. For GGE, the first sufficiently powered GWASs was the seminal study for the European EPICURE consortium, which discovered common variants at CHRM3 at 1q43, VRK2/FANCL at 2p16.1, ZEB2 at 2q22.3, PNPO at 17q21.32, and SCN1A at $2 \mathrm{q} 24.3$ as risk factors for GGE. ${ }^{37}$ This discovery required the joint power of more than 3,000 patients and 3,000 controls. A follow-up meta-analysis reinforced the association with VRK2/FANCL at 2p16.1, while calling the other association signals into question. ${ }^{38}$ In addition, the meta-analysis performed by the ILAE Consortium on Complex Epilepsies also emphasized the association with common SCN1A variants with all epilepsy subtypes combined that were joined in this analysis. These results suggest that genetic risk factors at previously unknown loci might give novel insight into GGE pathophysiology and that SCN1A variants confer risk along a spectrum of variants, ranging from the de novo dominant mutations in Dravet syndrome to the common variants predisposing to common epilepsies. It should be noted, however, that it is inherent in the technology of GWASs that the causative variant in this region cannot be pinned down through genetic studies. In fact, the variant with the strongest association signal is not within the SCN1A gene, but between the various genes of the sodium channel gene cluster on chromosome $2 .^{38}$ Regardless of these findings, the contribution of common variations in GGE seems too limited to a few prominent loci that confer relatively little risk.

\section{Next-Generation Sequencing}

With the limited availability of monogenic families and having exhausted the genetic potential of genome-wide studies of copy numbers and common variants, the field is currently turning to the emerging technologies of exome sequencing and genome sequencing to search for additional genetic risk factors in common genetic epilepsies. Although the field of gene identification in severe epileptic encephalopathies is currently on the upswing due to the increasing discovery of de novo mutations, this genetic paradigm is usually not invoked in patients with mild epilepsies. This is because the de novo events, frequently considered in severe disorders that limit an affected individual's capacity to reproduce, are unlikely to be found in common disorders. Accordingly, GGE genetics is largely limited to association studies, which present a perceptible challenge with next-generation sequencing technologies.

The first results from exome sequencing GGEs were largely negative. An association study on rare variants identified through exome sequencing in GGE that were followed up in more than 800 GGE patients and 1,830 controls found no "Goldilocks variants"-rare, but still relatively frequent genetic variants that predispose to GGE. ${ }^{39}$ However, largerscale studies probing the genetic architecture of GGE are currently underway, hopefully giving insight into the underlying biology through larger samples sizes.

\section{An Integrative View of GGE Genetics}

Despite the deeply convincing evidence for a genetic contribution in GGE through twin and family studies, the genetic architecture underlying these common epilepsies remains to be solved. In fact, as of 2015, gene discovery in GGE has even fallen behind the speed of gene identification in other epilepsies, demonstrating the complexity in pinning down heterogeneous rare risk factors, which-by exclusion-are believed to harbor most of the genetic risk. The decade-long search for genetic risk factors for GGE has resulted in several unexpected findings along the way, including the discovery of potentially treatable GLUT1-deficiency syndromes in atypical cases and the genetic overlap with other neurodevelopmental disorders through the identification of CNVs (-Table 1). Genome-wide association studies have highlighted common genetic risk factors: The association of variants close to SCN1A has emerged as a common theme in various epilepsies. Although these studies have successively chipped away at the genetic architecture of GGE, the bulk of the genetic risk remains unexplained. Large-scale exome or genome-sequencing studies will probably substantially fill this gap in the near future.

\section{References}

1 Jallon P, Loiseau P, Loiseau J. Newly diagnosed unprovoked epileptic seizures: presentation at diagnosis in CAROLE study. Coordination Active du Réseau Observatoire Longitudinal de l' Epilepsie. Epilepsia 2001;42(4):464-475

2 Berg AT, Berkovic SF, Brodie MJ, et al. Revised terminology and concepts for organization of seizures and epilepsies: report of the ILAE Commission on Classification and Terminology, 2005-2009. Epilepsia 2010;51(4):676-685

3 Peljto AL, Barker-Cummings C, Vasoli VM, et al. Familial risk of epilepsy: a population-based study. Brain 2014;137(Pt 3): 795-805 
4 Scheffer IE, Berkovic SF. Generalized epilepsy with febrile seizures plus. A genetic disorder with heterogeneous clinical phenotypes. Brain 1997;120(Pt 3):479-490

5 Berkovic SF, Howell RA, Hay DA, Hopper JL. Epilepsies in twins: genetics of the major epilepsy syndromes. Ann Neurol 1998;43(4): 435-445

6 Kjeldsen MJ, Kyvik KO, Christensen K, Friis ML. Genetic and environmental factors in epilepsy: a population-based study of 11900 Danish twin pairs. Epilepsy Res 2001;44(2-3):167-178

7 Escayg A, MacDonald BT, Meisler MH, et al. Mutations of SCN1A, encoding a neuronal sodium channel, in two families with GEFS+2. Nat Genet 2000;24(4):343-345

8 Wallace RH, Wang DW, Singh R, et al. Febrile seizures and generalized epilepsy associated with a mutation in the $\mathrm{Na}+-$ channel beta1 subunit gene SCN1B. Nat Genet 1998;19(4): 366-370

9 Wallace RH, Scheffer IE, Barnett S, et al. Neuronal sodium-channel alpha1-subunit mutations in generalized epilepsy with febrile seizures plus. Am J Hum Genet 2001;68(4):859-865

10 Harkin LA, Bowser DN, Dibbens LM, et al. Truncation of the GABA (A)-receptor gamma2 subunit in a family with generalized epilepsy with febrile seizures plus. Am J Hum Genet 2002;70(2):530-536

11 Baulac S, Huberfeld G, Gourfinkel-An I, et al. First genetic evidence of $G A B A(A)$ receptor dysfunction in epilepsy: a mutation in the gamma2-subunit gene. Nat Genet 2001;28(1):46-48

12 Wallace RH, Marini C, Petrou S, et al. Mutant GABA(A) receptor gamma2-subunit in childhood absence epilepsy and febrile seizures. Nat Genet 2001;28(1):49-52

13 Berkovic SF, Mulley JC, Scheffer IE, Petrou S. Human epilepsies: interaction of genetic and acquired factors. Trends Neurosci 2006; 29(7):391-397

14 Schubert J, Siekierska A, Langlois M, et al; EuroEPINOMICS RES Consortium. Mutations in STX1B, encoding a presynaptic protein, cause fever-associated epilepsy syndromes. Nat Genet 2014; 46(12):1327-1332

15 Cossette P, Liu L, Brisebois K, et al. Mutation of GABRA1 in an autosomal dominant form of juvenile myoclonic epilepsy. Nat Genet 2002;31(2):184-189

16 Suzuki T, Delgado-Escueta AV, Aguan K, et al. Mutations in EFHC1 cause juvenile myoclonic epilepsy. Nat Genet 2004;36(8):842-849

17 Subaran RL, Conte JM, Stewart WC, Greenberg DA. Pathogenic EFHC1 mutations are tolerated in healthy individuals dependent on reported ancestry. Epilepsia 2015;56(2):188-194

18 Cherepanova NS, Leslie E, Ferguson PJ, Bamshad MJ, Bassuk AG. Presence of epilepsy-associated variants in large exome databases. J Neurogenet 2013;27(1-2):1-4

19 Chen Y, Lu J, Pan H, et al. Association between genetic variation of CACNA1H and childhood absence epilepsy. Ann Neurol 2003; 54(2):239-243

20 Suls A, Mullen SA, Weber YG, et al. Early-onset absence epilepsy caused by mutations in the glucose transporter GLUT1. Ann Neurol 2009;66(3):415-419

21 Mullen SA, Marini C, Suls A, et al. Glucose transporter 1 deficiency as a treatable cause of myoclonic astatic epilepsy. Arch Neurol 2011;68(9):1152-1155
22 Seidner G, Alvarez MG, Yeh JI, et al. GLUT-1 deficiency syndrome caused by haploinsufficiency of the blood-brain barrier hexose carrier. Nat Genet 1998;18(2):188-191

23 Suls A, Dedeken P, Goffin K, et al. Paroxysmal exercise-induced dyskinesia and epilepsy is due to mutations in SLC2A1, encoding the glucose transporter GLUT1. Brain 2008;131(Pt 7):1831-1844

24 Striano P, Weber YG, Toliat MR, et al; EPICURE Consortium. GLUT1 mutations are a rare cause of familial idiopathic generalized epilepsy. Neurology 2012;78(8):557-562

25 Mefford HC, Eichler EE. Duplication hotspots, rare genomic disorders, and common disease. Curr Opin Genet Dev 2009;19(3): 196-204

26 Redon R, Ishikawa S, Fitch KR, et al. Global variation in copy number in the human genome. Nature 2006;444(7118):444-454

27 Helbig I, Mefford HC, Sharp AJ, et al. 15q13.3 microdeletions increase risk of idiopathic generalized epilepsy. Nat Genet 2009; 41(2):160-162

28 Dibbens LM, Mullen S, Helbig I, et al; EPICURE Consortium. Familial and sporadic 15q13.3 microdeletions in idiopathic generalized epilepsy: precedent for disorders with complex inheritance. Hum Mol Genet 2009;18(19):3626-3631

29 de Kovel CG, Trucks H, Helbig I, et al. Recurrent microdeletions at $15 q 11.2$ and 16p13.11 predispose to idiopathic generalized epilepsies. Brain 2010;133(Pt 1):23-32

30 Boone PM, Wiszniewski W, Lupski JR. Genomic medicine and neurological disease. Hum Genet 2011;130(1):103-121

31 Sharp AJ. Emerging themes and new challenges in defining the role of structural variation in human disease. Hum Mutat 2009; 30(2):135-144

32 Mullen SA, Carvill GL, Bellows S, et al. Copy number variants are frequent in genetic generalized epilepsy with intellectual disability. Neurology 2013;81(17):1507-1514

33 Carvill GL, Mefford HC. Microdeletion syndromes. Curr Opin Genet Dev 2013;23(3):232-239

34 Helbig I, Lowenstein DH. Genetics of the epilepsies: where are we and where are we going? Curr Opin Neurol 2013;26(2):179-185

35 Helbig I, Hodge SE, Ottman R. Familial cosegregation of rare genetic variants with disease in complex disorders. Eur J Hum Genet 2013;21(4):444-450

36 Manolio TA, Collins FS, Cox NJ, et al. Finding the missing heritability of complex diseases. Nature 2009;461(7265):747-753

37 Steffens M, Leu C, Ruppert AK, et al; EPICURE Consortium; EMINet Consortium. Genome-wide association analysis of genetic generalized epilepsies implicates susceptibility loci at 1q43, 2p16.1, 2q22.3 and 17q21.32. Hum Mol Genet 2012; 21(24):5359-5372

38 International League Against Epilepsy Consortium on Complex Epilepsies. Electronic address: epilepsy-austin@unimelb.edu.au. Genetic determinants of common epilepsies: a meta-analysis of genome-wide association studies. Lancet Neurol 2014;13(9): 893-903

39 Heinzen EL, Depondt C, Cavalleri GL, et al. Exome sequencing followed by large-scale genotyping fails to identify single rare variants of large effect in idiopathic generalized epilepsy. Am J Hum Genet 2012;91(2):293-302 